PROCEEDINGS OF THE

AMERICAN MATHEMATICAL SOCIETY

Volume 133, Number 10, Pages 2853-2863

S 0002-9939(05)07900-1

Article electronically published on March 29, 2005

\title{
CHARACTERIZATION OF BILINEAR SPACES WITH UNIMODULAR ISOMETRY GROUP
}

\author{
DRAGOMIR Ž. ĐOKOVIĆ AND FERNANDO SZECHTMAN
}

(Communicated by Jonathan I. Hall)

\begin{abstract}
We study finite-dimensional bilinear spaces and their isometry groups. To each bilinear space $V$ we associate two canonical filtrations, which yield structural results on $V$. Prominent among these is an explicit formula for the number of indecomposable and degenerate blocks of $V$ of a given dimension. Equipped with this material, we proceed to characterize those bilinear spaces whose isometry group is contained in the special linear group. This characterization can easily be implemented in practice by means of an algorithm. As an application, we determine the real $n$-by- $n$ matrices whose congruence class is disconnected.
\end{abstract}

\section{INTRODUCTION}

A bilinear space over a field $F$ is a pair $(V, \varphi)$, where $V$ is a finite-dimensional $F$-vector space and $\varphi: V \times V \rightarrow F$ a bilinear form. The main problem concerning bilinear spaces is to determine when two of them are equivalent, i.e. they afford a linear isomorphism preserving the underlying forms. There is a structure theory developed around this problem, with contributions by J. Williamson [19], G.E. Wall 17], C. Riehm [14, P. Gabriel [8] and R. Scharlau [15]. The principal result of this theory is the reduction of the equivalence problem of bilinear spaces to classical problems in linear algebra, such as the similarity of matrices and the equivalence of quadratic and hermitian forms. More recently, a degree of attention has been focused on finding representatives or their numbers for the Gram matrices of bilinear spaces, under certain limitations either on the field or the size of the matrix. See for instance 9, 18, 12, 2, 3, 4.

Of all bilinear spaces, those whose underlying form is symmetric are by far the ones most widely considered, with an extensive theory surrounding them from the algebraic, geometric or arithmetic point of view (see e.g. [13, 16] and their bibliographies). The comparative neglect suffered by general bilinear spaces probably explains why certain basic results took so long to be established. For example, the fact that a square matrix over an arbitrary field is congruent to its transpose was not proved until 1980 by R. Gow [10] for invertible matrices and shortly afterwards by Ballantine and Yip [1] in general.

A more deeply marked difference occurs with the isometry group associated to a bilinear space. On one hand one has the classical linear groups arising as isometry

Received by the editors November 7, 2003 and, in revised form, May 27, 2004.

2000 Mathematics Subject Classification. Primary 15A63.

The first author was supported in part by the NSERC Grant A-5285. 
groups of zero, alternating or symmetric forms -and subjected to detailed scrutiny (see e.g. [5, 6, 11]) - and on the other the general isometry groups of which virtually nothing has been written.

The present paper aims at contributing to the structure theory of bilinear spaces, with an eye on application to the study of the corresponding isometry groups. One such application is dealt with in this paper. Indeed, one of our main results is a characterization of bilinear spaces whose isometry group is unimodular, i.e., contained in the special linear group.

We refer to a bilinear space as totally degenerate if every non-zero orthogonal direct summand of it is degenerate. Any bilinear space, $V$ for short, decomposes as the orthogonal direct sum $V=V_{\text {ndeg }} \perp V_{\text {tdeg }}$, where $V_{\text {ndeg }}$ is non-degenerate and $V_{\text {tdeg }}$ is totally degenerate. Both $V_{\text {ndeg }}$ and $V_{\text {tdeg }}$ are unique only up to equivalence. This result is due to P. Gabriel [8]. One of our most interesting results is a formula for the number $m(r)$ of indecomposable degenerate spaces of dimension $r$ (they are all equivalent to each other) present in a given orthogonal decomposition of $V$ into indecomposables. Indeed,

$$
m(r)=(-1)^{r}\left(l_{r-2}+l_{r-1}-l_{r}-l_{r+1}\right), \quad r \geq 1,
$$

where $l_{-1}=0, l_{i}=\operatorname{dim} L^{i}(V), i \geq 0$, and $L^{i}$ is the $i$-iterate of the operator $L$, which associates to each subspace of $V$ its left orthogonal complement. By means of $L$ and the analogous operator $R$ from the right we obtain structural invariants of $V$, which are somewhat technical to describe at this point.

In general, neither $V_{\text {ndeg }}$ nor $V_{\text {tdeg }}$ is stable under the isometry group of $V$. However, the asymmetry, say $\sigma$, of $V_{\text {ndeg }}$, as defined in [14], is uniquely determined by $V$ up to similarity. It is a linear operator that somehow measures how far $\varphi$ is from being symmetric on $V_{\text {ndeg }}$.

With this notation, we prove that the isometry group $G$ of $V$ is unimodular if and only if $m(r)=0$ for all odd $r$ and $\sigma$ has no unipotent Jordan blocks of odd size. This holds for all fields $F$ of characteristic not 2 . If $\operatorname{char} F=2$ but $|F|>2$, then $G$ is unimodular precisely when every orthogonal direct summand of $V_{\text {tdeg }}$ is even dimensional. When $|F|=2$ the group $G$ is always unimodular.

As an application, we show that a real square matrix $A$ has disconnected congruence class if and only if $A$ has no direct summands of odd size (under congruence transformations).

We are indebted to a referee for several corrections, and for suggestions to clarify some arguments in the proofs of Theorems 3.2 and 4.6 .

\section{Notation AND PRELIMINARIES}

Let $(V, \varphi)$ be a bilinear space. Explicit reference to $\varphi$ will be omitted when no confusion is possible. We shall often write $\langle v, w\rangle$ instead of $\varphi(v, w)$.

If $U$ is a subspace of $V$, then $U$ becomes a bilinear space by restricting $\varphi$ to $U \times U$. We write $V=U \perp W$ if $V=U \oplus W$ and $\langle U, W\rangle=\langle W, U\rangle=0$. In this case we refer to $U$ and $W$ as orthogonal summands of $V$. A bilinear space is indecomposable if it lacks proper non-zero orthogonal summands. An isometry between bilinear spaces is a linear isomorphism which preserves the underlying forms, in which case the spaces are said to be equivalent. The group of all isometries of $(V, \varphi)$ will be denoted by $\operatorname{Iso}(V, \varphi)$. 
For a subspace $U$ of $V$, let

$$
L(U)=\{v \in V \mid\langle v, U\rangle=0\}, \quad R(U)=\{v \in V \mid\langle U, v\rangle=0\} .
$$

Here $L(V)$ and $R(V)$ are the left and right radicals of $V$, and $L(V) \cap R(V)$ is the radical of $V$. We have $\operatorname{dim} L(V)=\operatorname{dim} R(V)$, and we say that $V$ is non-degenerate whenever this number is 0 . Otherwise $V$ is degenerate. We shall say that $V$ is totally degenerate if all non-zero orthogonal summands of $V$ are degenerate.

We may view $L$ and $R$ as operators which assign to each subspace of $V$ its left and right orthogonal complements, respectively. We may compound these operators, denoting by $L^{i}$ and $R^{i}$ their respective $i$-th iterates. By convention, $L^{0}$ and $R^{0}$ are the identity operators. When more than one bilinear space is present, we shall write $L_{V}$ and $R_{V}$ for $L$ and $R$. For instance, $R_{V}=L_{V^{\prime}}$, where $V^{\prime}$ is the transpose of the bilinear space $V$.

For $r \geq 1$, denote by $J_{r}$ the nilpotent lower Jordan block of size $r$. Thus

$$
J_{1}=(0), \quad J_{2}=\left(\begin{array}{cc}
0 & 0 \\
1 & 0
\end{array}\right), \quad J_{3}=\left(\begin{array}{lll}
0 & 0 & 0 \\
1 & 0 & 0 \\
0 & 1 & 0
\end{array}\right), \ldots
$$

Write $N_{r}$ for a bilinear space whose underlying form has matrix $J_{r}$ relative to some basis. We shall refer to the bilinear space $N_{r}$ as a Gabriel block and to $r$ as its size. We point out that, from the point of view of the theory of matrix pencils, there is another natural choice for a basis of $N_{r}$. The corresponding matrices are given explicitly in [18].

We refer the reader to [7, 18] for the following formulation of a theorem due to P. Gabriel 8 .

Theorem 2.1. Let $V$ be a bilinear space over $F$. Then

(a) $V=V_{\text {tdeg }} \perp V_{\text {ndeg }}$, where $V_{\text {tdeg }}$ is the orthogonal direct sum of Gabriel blocks and $V_{\text {ndeg }}$ is non-degenerate (either of them possibly 0 ).

(b) The sizes and multiplicities of the Gabriel blocks appearing in $V_{\mathrm{tdeg}}$ are uniquely determined by $V$.

(c) The equivalence class of $V_{\text {ndeg }}$ is uniquely determined by $V$.

(d) Up to equivalence, the only indecomposable and degenerate bilinear space of dimension $r \geq 1$ is $N_{r}$.

We refer to $V_{\text {tdeg }}$ and $V_{\text {ndeg }}$ as the totally degenerate and non-degenerate parts of $V$, respectively (this defines them up to equivalence only). We may write $V_{\mathrm{tdeg}}=$ $V_{\text {even }} \perp V_{\text {odd }}$, where $V_{\text {even }}$ (resp. $V_{\text {odd }}$ ) is the orthogonal direct sum of Gabriel blocks of even (resp. odd) size. We refer to them as the even and odd parts of $V$ (again, they are defined up to equivalence only).

We shall say that a sequence $\left(U_{i}\right)_{i \geq 0}$ of subspaces of $V$ is nested if

$$
U_{1} \subseteq U_{3} \subseteq U_{5} \subseteq \cdots \subseteq U_{4} \subseteq U_{2} \subseteq U_{0}=V .
$$

The subsequences $\left(U_{2 i+1}\right)_{i \geq 0}$ and $\left(U_{2 i}\right)_{i \geq 0}$ stabilize. We shall refer to the subspaces $U_{\infty}$ and $U^{\infty}$ in which $\left(U_{2 i+1}\right)_{i \geq 0}$ and $\left(U_{2 i}\right)_{i \geq 0}$ stabilize as the lower and upper limits of $\left(U_{i}\right)_{i \geq 0}$, respectively. Clearly the former is contained in the latter. If these limits coincide, then $\left(U_{i}\right)_{i \geq 0}$ is said to be convergent.

As we shall see, the sequences $\left(L^{i}(V)\right)_{i \geq 0}$ and $\left(R^{i}(V)\right)_{i \geq 0}$ are nested, yielding canonical filtrations for $V$. We write $L_{\infty}(V)$ and $L^{\infty}(V)$ (resp. $R_{\infty}(V)$ and $R^{\infty}(V)$ ) for the lower and upper limits of $\left(L^{i}(V)\right)_{i \geq 0}$ (resp. $\left.\left(R^{i}(V)\right)_{i \geq 0}\right)$.

Given vectors $v_{1}, \ldots, v_{s}$ of a vector space, their span will be denoted by $\left[v_{1}, \ldots, v_{s}\right]$. 


\section{Structural Results}

In this section we study the structure of arbitrary bilinear spaces and pay special attention to some important orthogonal direct decompositions of these spaces.

Theorem 3.1. Let $N_{r}$ be a Gabriel block of size $r$. Then

(a) The sequences $\left(L^{i}\left(N_{r}\right)\right)_{i \geq 0}$ and $\left(R^{i}\left(N_{r}\right)\right)_{i \geq 0}$ are nested and convergent.

(b) If $r$ is even, then $N_{r}=L^{\infty}\left(N_{r}\right) \oplus R^{\infty}\left(N_{r}\right)$.

(c) If $r$ is odd, then $L^{\infty}\left(N_{r}\right)=R^{\infty}\left(N_{r}\right)$.

(d) If $r$ is even, then $N_{r}=L^{\infty}\left(N_{r}\right) \oplus R^{\infty}\left(N_{r}\right)$ is the only decomposition of $N_{r}$ as the direct sum of two totally isotropic subspaces.

(e) If $r$ is odd, then $L^{\infty}\left(N_{r}\right)=R^{\infty}\left(N_{r}\right)$ is the only totally isotropic subspace of $N_{r}$ of maximum dimension.

Proof. Let $\left\{e_{1}, \ldots, e_{r}\right\}$ be a basis for $N_{r}$, relative to which the underlying form has matrix $J_{r}$. Then

$$
\begin{aligned}
& L^{1}\left(N_{r}\right)=\left[e_{1}\right], \quad L^{2}\left(N_{r}\right)=\left[e_{1}, e_{3}, e_{4}, \ldots, e_{r}\right] \\
& L^{3}\left(N_{r}\right)=\left[e_{1}, e_{3}\right], \quad L^{4}\left(N_{r}\right)=\left[e_{1}, e_{3}, e_{5}, e_{6}, \ldots, e_{r}\right], \ldots
\end{aligned}
$$

and

$$
\begin{aligned}
& R^{1}\left(N_{r}\right)=\left[e_{r}\right], \quad R^{2}\left(N_{r}\right)=\left[e_{r}, e_{r-2}, e_{r-3}, \ldots, e_{1}\right] \\
& R^{3}\left(N_{r}\right)=\left[e_{r}, e_{r-2}\right], \quad R^{4}\left(N_{r}\right)=\left[e_{r}, e_{r-2}, e_{r-4}, e_{r-5}, \ldots, e_{1}\right], \ldots
\end{aligned}
$$

From these identities we easily obtain (a), (b) and (c). It is also clear that $L^{\infty}\left(N_{r}\right)$ and $R^{\infty}\left(N_{r}\right)$ are totally isotropic regardless of the parity of $r$, and that the maximum dimension for a totally isotropic subspace of $N_{r}$ for $r$ odd is $(r+1) / 2$. This is attained by $L^{\infty}\left(N_{r}\right)=R^{\infty}\left(N_{r}\right)$.

We next show (d) by induction on $r$. If $r=2$, then $\left[e_{1}\right]$ and $\left[e_{2}\right]$ are the only totally isotropic subspaces of $N_{r}$, whence the result holds. Suppose $r \geq 4$ and let $N_{r}=U \oplus W$ be a decomposition of $N_{r}$ into totally isotropic subspaces. We easily see that

$$
L\left(N_{r}\right)=U \cap L\left(N_{r}\right) \oplus W \cap L\left(N_{r}\right) .
$$

As $L\left(N_{r}\right)$ is one dimensional, either $U$ or $W$ contains $L\left(N_{r}\right)$. Since we also have

$$
L(U)=U+L\left(N_{r}\right) \text { and } L(W)=W+L\left(N_{r}\right),
$$

we infer that either $U=L(U)$ or $W=L(W)$ (both possibilities cannot occur because $L\left(N_{r}\right)=L(U) \cap L(W)$ is not zero). Without loss of generality, we may assume that $L\left(N_{r}\right) \subseteq U$ and $U=L(U)$.

Since $L\left(N_{r}\right)$ is contained in the radical of $L^{2}\left(N_{r}\right)$, we may consider the quotient bilinear space $L^{2}\left(N_{r}\right) / L\left(N_{r}\right)$, which is easily seen to be equivalent to $N_{r-2}$ (cf. Lemma 3.1 of [7]). From the above discussion we infer that $U \subseteq L^{2}\left(N_{r}\right)$, whence $U / L\left(N_{r}\right)$ is a totally isotropic subspace of $L^{2}\left(N_{r}\right) / L\left(N_{r}\right)$. In fact, we have the decomposition

$$
L^{2}\left(N_{r}\right) / L\left(N_{r}\right)=U / L\left(N_{r}\right) \oplus\left(\left(W^{\prime} \oplus L\left(N_{r}\right)\right) / L\left(N_{r}\right)\right)
$$

into totally isotropic subspaces, where $W^{\prime}=L^{2}\left(N_{r}\right) \cap W$. However, we also have the decomposition

$$
L^{2}\left(N_{r}\right) / L\left(N_{r}\right)=L^{\infty}\left(N_{r}\right) / L\left(N_{r}\right) \oplus\left(\left(\left[e_{4}, e_{6}, \ldots, e_{r}\right] \oplus L\left(N_{r}\right)\right) / L\left(N_{r}\right)\right) .
$$


It follows by induction that $U=L^{\infty}\left(N_{r}\right)$ or $U=\left[e_{1}, e_{4}, e_{6}, \ldots, e_{r}\right]$. The latter is impossible since $e_{3} \in L\left(\left[e_{1}, e_{4}, e_{6}, \ldots, e_{r}\right]\right)$, whereas $U=L(U)$. This establishes $U=L^{\infty}\left(N_{r}\right)$.

Similarly, either $U$ or $W$ must contain $R\left(N_{r}\right)$, and whichever contains it must equal $R^{\infty}\left(N_{r}\right)$. Since $e_{r} \notin U$, it follows that $W=R^{\infty}\left(N_{r}\right)$.

We finally prove (e) by induction on $r$. The case $r=1$ is clear. Suppose $r \geq 3$ and let $U$ be a totally isotropic subspace of $N_{r}$ of maximum dimension, i.e., $(r+1) / 2$. As $U$ is totally isotropic, we have $U \subseteq L(U)$. On the other hand since the canonical linear map $L(U) \rightarrow\left(N_{r} / U\right)^{*}$ has the 1-dimensional space $L\left(N_{r}\right)$ as its kernel, we have

$$
\operatorname{dim} L(U)-1 \leq \frac{r-1}{2} .
$$

Therefore $U=L(U)$ and $L\left(N_{r}\right) \subseteq U$. Thus $U / L\left(N_{r}\right)$ is a totally isotropic subspace of $L^{2}\left(N_{r}\right) / L\left(N_{r}\right)$. Since the latter is equivalent to $N_{r-2}$, it follows by induction that $U=L^{\infty}\left(N_{r}\right)$.

Theorem 3.2. Let $V$ be a bilinear space over $F$. Then

(a) The sequences $\left(L^{i}(V)\right)_{i \geq 0}$ and $\left(R^{i}(V)\right)_{i \geq 0}$ are nested. They converge if and only if $V$ is totally degenerate. To simplify the notation, set $V_{\infty}=L_{\infty}(V) \cap R_{\infty}(V)$, $V^{\infty}=L^{\infty}(V) \cap R^{\infty}(V),{ }_{\infty} V=L_{\infty}(V)+R_{\infty}(V)$ and ${ }^{\infty} V=L^{\infty}(V)+R^{\infty}(V)$.

(b) $V_{\infty}$ is the radical of the following bilinear spaces: $V_{\infty}, \infty V, V^{\infty}$ and ${ }^{\infty} V$.

(c) In general none of the subspaces $V_{\text {even }}, V_{\text {odd }}, V_{\text {tdeg }}$ and $V_{\text {ndeg }}$ is uniquely determined by $V$. However, $V_{\text {odd }}$ has a unique totally isotropic subspace of maximum dimension, and this is uniquely determined by $V$. In fact, it is equal to $V_{\infty}$. Moreover, $V_{\text {even }} \perp V_{\infty}, V_{\text {ndeg }} \perp V_{\infty}$ and $V_{\text {ndeg }} \perp V_{\text {even }} \perp V_{\infty}$ are uniquely determined by $V$, being respectively equal to $\infty_{\infty} V, V^{\infty}$, and ${ }^{\infty} V$.

(d) If $V_{\mathrm{odd}}=0$, then $V_{\mathrm{tdeg}}$ and $V_{\mathrm{ndeg}}$ are uniquely determined by $V$. In fact, we have $V_{\mathrm{tdeg}}={ }_{\infty} V$ and $V_{\mathrm{ndeg}}=V^{\infty}$.

(e) We have the following equivalences between bilinear spaces:

$$
\begin{aligned}
& \infty V / V_{\infty} \cong V_{\text {even }}, \\
& V^{\infty} / V_{\infty} \cong V_{\text {ndeg }} \quad \text { and } \\
& { }^{\infty} V / V_{\infty} \cong V_{\text {even }} \perp V_{\text {ndeg }} .
\end{aligned}
$$

Proof. We begin by observing that if $V=U \perp W$ and $V^{\prime}=U^{\prime} \perp W^{\prime}$, with $U^{\prime} \subseteq U$ and $W^{\prime} \subseteq W$, then

$$
L_{V}\left(V^{\prime}\right)=L_{U}\left(U^{\prime}\right) \perp L_{W}\left(W^{\prime}\right) .
$$

Note further that if $W$ is non-degenerate, then $L_{W}(W)=0$ and $L_{W}^{2}(W)=W$. Applying these remarks to the decomposition $V=V_{\text {tdeg }} \perp V_{\text {ndeg }}$ of Theorem 2.1 . and making use of Theorem 3.1 we immediately obtain (a) and (b).

The first statement from (c) is easily seen by examples. For instance, let $V$ be 4-dimensional with basis $\left\{e_{1}, e_{2}, e_{3}, e_{4}\right\}$ and let

$$
\varphi\left(e_{2}, e_{1}\right)=\varphi\left(e_{3}, e_{2}\right)=\varphi\left(e_{4}, e_{4}\right)=1,
$$

while all other $\varphi\left(e_{i}, e_{j}\right)=0$. Let $\alpha, \beta \in F$ be arbitrary scalars. Then we can take

$$
V_{\mathrm{tdeg}}=V_{\mathrm{odd}}=\left[e_{1}, e_{3}, \alpha e_{1}+e_{2}-\left(\alpha+\beta^{2}\right) e_{3}-\beta e_{4}\right], \quad V_{\mathrm{ndeg}}=\left[\beta e_{1}+\beta e_{3}+e_{4}\right] .
$$

This shows the non-uniqueness of $V_{\text {ndeg }}, V_{\text {odd }}$, and $V_{\text {tdeg }}$.

In regards to the second, we may assume that $V=V_{\text {odd }}$. The proof is similar to that of part (e) of the previous theorem. We proceed by induction on the 
dimension of $V$. Let $V=V_{1} \perp V_{2} \perp \ldots \perp V_{k}$, where $V_{i}$ is a Gabriel block of size $r_{i}=2 s_{i}+1$. Then $V_{\infty}$ has dimension $d:=\sum\left(s_{i}+1\right)$. Let $U$ be a totally isotropic subspace of $V$ of maximum dimension, and so $\operatorname{dim} U \geq d$. Since the canonical linear map $L(U) \rightarrow(V / U)^{*}$ has the $k$-dimensional space $L(V)$ as its kernel, we have $\operatorname{dim} L(U)-k \leq \sum s_{i}$, i.e., $\operatorname{dim} L(U) \leq \operatorname{dim} U$. As $U \subseteq L(U)$, it follows that $U=L(U)$ and $L(V) \subseteq U$. Thus $U / L(V)$ is a totally isotropic subspace of $L^{2}(V) / L(V)$. Since the latter is equivalent to

$$
\underset{r_{i}>1}{\stackrel{1}{~}} N_{r_{i}-2},
$$

we can use induction to finish the proof.

The remaining part of (c) is clear, and it is easy to verify the assertions (d) and (e).

For $i \geq 0$, let us write $l_{i}$ for the dimension of $L^{i}(V)$. For convenience, we set $l_{-1}=0$. For $r \geq 1$ we shall write $m(r)$ (resp. $n(r)$ ) for the number of Gabriel blocks of size $r$ (resp. $\geq r$ ) appearing in a given orthogonal decomposition of $V$.

Theorem 3.3. Let $V$ be a bilinear space over $F$. Then

$$
m(r)=(-1)^{r}\left(l_{r-2}+l_{r-1}-l_{r}-l_{r+1}\right), \quad r \geq 1 .
$$

Proof. The formulae at the very beginning of the proofs of Theorems 3.1 and 3.2 show

$$
\begin{aligned}
l_{0} & =\operatorname{dim} V, \\
l_{1}-l_{-1} & =n(1), \\
l_{0}-l_{2} & =n(2), \\
l_{3}-l_{1} & =n(3), \\
l_{2}-l_{4} & =n(4), \text { etc. }
\end{aligned}
$$

Since $m(r)=n(r)-n(r+1)$ for all $r \geq 1$, the result follows.

Combining Theorems 3.2 (e) and 3.3 yields another proof of parts (b) and (c) of Theorem 2.1 (note that part (a) follows from the other parts).

\section{WHEN IS THE ISOMETRY GROUP UNIMODULAR?}

In this section we consider a bilinear space $(V, \varphi)$ over a field $F$ and its isometry group $G=\operatorname{Iso}(V, \varphi)$. We say that $G$ is unimodular if it is contained in $\operatorname{SL}(V)$. We shall find necessary and sufficient conditions for $G$ to be unimodular. If $|F|=2$, then $\mathrm{SL}(V)=\mathrm{GL}(V)$ and $G \subseteq \mathrm{SL}(V)$ holds trivially. Thus we shall assume that $|F|>2$.

Lemma 4.1. If $G$ is unimodular and $|F|>2$, then $V_{\text {odd }}=0$.

Proof. Assume that $V=N_{r} \perp W$ with $r$ odd. Then $\operatorname{Iso}\left(N_{r}\right)$ is a subgroup of $G$. Choose a basis of $N_{r}$ in which its bilinear form has matrix $J_{r}$. If $a \in F$ and $a \neq 0,1$, then

$$
\operatorname{diag}\left(a, a^{-1}, a, a^{-1}, \ldots, a^{-1}, a\right) \in \operatorname{Iso}\left(N_{r}\right)
$$

has determinant $a$. Hence $G$ is not contained in $\operatorname{SL}(V)$. 
Let us make two trivial but useful observations. First, if $V$ is non-degenerate, then every $g \in G$ has determinant \pm 1 . In particular, when the characteristic is 2 , $G$ is unimodular. Second, we have

$$
\operatorname{Iso}(V, \varphi)=\operatorname{Iso}\left(V, \varphi^{\prime}\right) \subseteq \operatorname{Iso}\left(V, \varphi-\varphi^{\prime}\right) .
$$

This is useful because, when $\varphi-\varphi^{\prime}$ is non-degenerate, we have $\operatorname{Iso}\left(V, \varphi-\varphi^{\prime}\right) \subseteq$ $\operatorname{SL}(V)$. We refer to $\varphi-\varphi^{\prime}$ as the alternating part of $(V, \varphi)$.

The characteristic 2 case is completely covered by the following theorem.

Theorem 4.2. Assume that $|F|>2$. Then $G$ is unimodular if and only if $V_{\text {odd }}=0$ and $\operatorname{Iso}\left(V_{\mathrm{ndeg}}\right)$ is unimodular. If $F$ has characteristic 2 , then the second condition is automatically satisfied.

Proof. The first condition is necessary by Lemma 4.1

Assume that the first condition is satisfied. Then by Theorem $3.2(\mathrm{~d})$ we have $V=V_{\text {even }} \perp V_{\text {ndeg, }}$, where the summands are uniquely determined by $V$. Hence $G$ is the direct product of $\operatorname{Iso}\left(V_{\text {ndeg }}\right)$ and $\operatorname{Iso}\left(V_{\text {even }}\right)$. The alternating part of $V_{\text {even }}$ is non-degenerate, since that is the case for $N_{r}$ with $r$ even. Therefore by applying (4.1) to $V_{\text {even, }}$, we see that the latter group is unimodular. Hence, $G$ is unimodular if and only if so is $\operatorname{Iso}\left(V_{\text {ndeg }}\right)$.

The second assertion follows from the first observation above.

We have reduced the problem of unimodularity of $G$ to the non-degenerate case. For the remainder of this section we assume that the characteristic of $F$ is not 2 and that $V$ is non-degenerate. Following Riehm [14, we define the asymmetry of $(V, \varphi)$ as the linear operator $\sigma \in \mathrm{GL}(V)$ such that $\varphi^{\prime}(v, w)=\varphi(v, \sigma(w))$ for all $v, w \in V$. We view $V$ as a module over $F[t]$ via the action of $\sigma$. For an irreducible polynomial $p \in F[t]$, let $V_{p}$ denote the primary component of $V$ associated to $p$. Write $V_{\text {unip }}=V_{t-1}$.

Lemma 4.3. Iso $(V)$ is unimodular if and only if $\operatorname{Iso}\left(V_{\text {unip }}\right)$ is unimodular.

Proof. By 14, Proposition 3], we have $V=V_{\text {unip }} \perp W$, where both summands are uniquely determined by $V$. Hence $G$ is the direct product of $\operatorname{Iso}\left(V_{\text {unip }}\right)$ and $\operatorname{Iso}(W)$. Now $\varphi(v, w)-\varphi^{\prime}(v, w)=\varphi(v, w-\sigma(w))$, with $\left.\varphi\right|_{W \times W}$ non-degenerate and $\left.(1-\sigma)\right|_{W}$ invertible. Therefore $\left.\left(\varphi-\varphi^{\prime}\right)\right|_{W \times W}$ is non-degenerate. Hence by applying (4.1) to $W$, we conclude that $\operatorname{Iso}(W)$ is unimodular. Thus the result follows.

Suppose $V$ has an odd-dimensional orthogonal summand $W$. Then $-I_{W}$ can be extended to an isometry of $V$ with determinant -1 , so $V$ is not unimodular. We next derive an equivalent condition to the existence of such $W$ in terms of the elementary divisors of $V_{\text {unip }}$.

Lemma 4.4. Let $\varepsilon= \pm 1$. Suppose $V=V_{t-\varepsilon}$ and that $V$ is a free module over $F[t] /(t-\varepsilon)^{s}$ for some $s \geq 1$. Let $\pi=\sigma-\sigma^{-1}$ and $\bar{V}=V / \pi V$. Define $g_{s}: \bar{V} \times \bar{V} \rightarrow F$ by the formula

$$
g_{s}(\bar{u}, \bar{v})=\varphi\left(\pi^{s-1} u, v\right), \quad u, v \in V,
$$

where $\bar{u}=u+\pi V$ and $\bar{v}=v+\pi V$. Then

(a) $g_{s}$ is a well-defined non-degenerate bilinear form.

(b) $g_{s}$ has scalar asymmetry $(-1)^{s-1} \varepsilon$.

(c) $\operatorname{dim} V=s \cdot \operatorname{dim} \bar{V}$. 
Proof. The first two conditions are explained by [14, pp. 50, 53], while the third is clear.

Lemma 4.5. $V$ has an odd-dimensional orthogonal summand if and only if $V$ has an elementary divisor of the form $(t-1)^{s}$ with $s$ odd.

Proof. Suppose $V$ has an elementary divisor of the form $(t-1)^{s}$ with $s$ odd. Due to [14. Corollary to Proposition 3 and Lemma 6] we may assume that $V=V_{t-1}$ is a free module over $F[t] /(t-1)^{s}$. Construct the non-degenerate symmetric bilinear form $g_{s}$ of Lemma 4.4 Since $F$ has characteristic not 2, we infer that $g_{s}$ has a nonisotropic vector, i.e., there is a $v \in V$ such that $\varphi\left(\pi^{s-1} v, v\right) \neq 0$. It follows that the $F[t]$-submodule, say $W$, of $V$ generated by $v$ is non-degenerate and has dimension $s$. We conclude from [14, page 47] that $W$ is an odd-dimensional orthogonal summand of $V$.

Suppose conversely that $W$ is an odd-dimensional orthogonal summand of $V$. We may assume that $W=V$ is indecomposable. By [14 section 1] the minimal polynomial of $\sigma$ is a power of an irreducible monic polynomial $p(t)$ of odd degree. By [14, Proposition 1], we have $p(t)=t \pm 1$. By [14, Lemma 6 ], $V$ is a free module over $F[t] /(t \pm 1)^{s}$ for some odd $s$. Now $p(t)$ cannot equal $t+1$ due to Lemma 4.4. since skew-symmetric forms on odd-dimensional vector spaces over a field of characteristic not 2 do not exist. Thus $(t-1)^{s}$ is an elementary divisor of $V$.

We shall now prove the main result of this section.

Theorem 4.6. Let $(V, \varphi)$ be a bilinear space over a field $F$ of characteristic not 2 . Then $G=\operatorname{Iso}(V, \varphi)$ is unimodular if and only if $V_{\mathrm{odd}}=0$ and the asymmetry $\sigma$ of $V_{\text {ndeg }}$ has no elementary divisor $(t-1)^{d}$ with $d$ odd.

Proof. Necessity follows from Lemmas 4.1 and 4.5. To prove sufficiency we may assume by Theorem 4.2 and Lemma 4.3 that $V=V_{\text {ndeg }}=V_{\text {unip. }}$. Consider the nilpotent operator $\pi=\sigma-\sigma^{-1}$ and the associated increasing filtration:

$$
0=V(0) \subset V(1) \subset \cdots \subset V(t-1) \subset V(t)=V,
$$

where $V(s)=\operatorname{ker}\left(\pi^{s}\right)$ for each $s$. These are $G$-modules since $\sigma$ is in the center of $G$. We have $t=2 r$ by our hypothesis on $\sigma$.

On the $G$-module $M(s)=V(s) /(V(s-1)+\pi V(s+1)), s \geq 1$, consider the non-degenerate bilinear form $h_{s}$ of [14 p. 54]. Thus

$$
h_{s}(\bar{v}, \bar{w})=\varphi\left(\pi^{s-1} v, w\right), \quad v, w \in V(s)
$$

where, e.g., $\bar{v}$ is the coset of $v$ in $M(s)$. Recall that symplectic transformations have determinant 1 . Since $h_{s}$ is $G$-invariant and has scalar asymmetry $(-1)^{s-1}$, the action of $G$ on each $M(2 s)$ must be unimodular $(*)$.

Let $N(s)=V(s) / V(s-1)$ and $P(s)=(V(s-1)+\pi V(s+1)) / V(s-1)$, which are $G$-modules for $s \geq 1$. Note that $P(s) \subseteq N(s)$ with $N(s) / P(s) \cong M(s)$ as $G$ modules. From [14, formula (4)] and our hypothesis on $\sigma$ we see that $P(s)=N(s)$ for odd $s$. Let $f: N(s+1) \rightarrow P(s)$ be defined by $f(v+V(s))=\pi v+V(s-1)$, $v \in V(s+1)$. This is a well-defined epimorphism of $G$-modules, and we see from [14, formula (4)] that $\operatorname{dim} N(s+1)=\operatorname{dim} P(s)$, whence $N(s+1) \cong P(s)$. If $s$ is odd, this and $P(s)=N(s)$ yield $N(s) \cong N(s+1)$.

Note that the $N(s), 1 \leq s \leq 2 r$, are the factors of the normal series (4.2) of $V$, so it suffices to show that $G$ is unimodular on each $N(s)$. We do this by induction on $s$. If $s=2 r$, it is easy to check that $N(2 r)=M(2 r)$, so $G$ is unimodular by $(*)$. 
So assume that $s<2 r$ and that $G$ is unimodular on $N(k)$ for all $k, s<k \leq 2 r$. If $s$ is odd, then $N(s) \cong N(s+1)$ as $G$-modules, so $G$ is unimodular on $N(s)$ by the induction hypothesis. Suppose $s$ is even. Because of the exact sequence

$$
0 \rightarrow P(s) \rightarrow N(s) \rightarrow M(s) \rightarrow 0
$$

of $G$-modules and (*), it suffices to show that $G$ is unimodular on $P(s)$, and this follows from $P(s) \cong N(s+1)$.

Corollary 4.7. Let $(V, \varphi)$ be a bilinear space over a field $F$ of characteristic not 2. Then $G$ is unimodular if and only if $(V, \varphi)$ has no orthogonal summands of odd dimension.

The main theorem can be easily applied in practice. Indeed a bilinear space $(V, \varphi)$ is given by an $n$-by- $n$ matrix over $F$, where $n=\operatorname{dim} V$. Then it is easy to write a program (say in Maple) to compute the sequences $\left(L^{i}(V)\right)_{i \geq 0}$ and $\left(R^{i}(V)\right)_{i \geq 0}$. Hence by using Theorem 3.3 we can find the multiplicities $m(r)$ and verify whether or not $V_{\text {odd }}=0$. Next we can use Theorem 3.2 (e) to compute the matrix of $V_{\text {ndeg }}$ and its asymmetry $\sigma$. Finally one can check whether or not $\sigma$ has an elementary divisor $(t-1)^{d}$ with $d$ odd.

\section{An APPLiCATion}

Suppose here that the field $F$ is arbitrary and that $V$ is a finite-dimensional $F$-vector space. Write $B(V)$ for the space of all bilinear forms on $V$. There is a canonical left action of $\mathrm{GL}(V)$ on $B(V)$ such that the stabilizer of $\varphi \in B(V)$ equals $\operatorname{Iso}(V, \varphi)$. As usual, we have a bijection between the space of left cosets $\operatorname{GL}(V) / \operatorname{Iso}(V, \varphi)$ and the orbit $O_{\varphi}$ of $\varphi$. In the real case this bijection is a homeomorphism (see 20, Theorems 3.1.3 and 2.1.14]). Hence $O_{\varphi}$ is either connected or it has two connected components, depending on whether $\operatorname{Iso}(V, \varphi)$ contains operators with negative determinant or not, respectively. It is natural to inquire under what conditions $O_{\varphi}$ will actually be disconnected. As a corollary of the results in the previous section, we obtain the following characterization, written in matrix terms.

Theorem 5.1. Let $A \in M_{n}(\mathbf{R})$ and $O_{A}=\left\{X^{\prime} A X: X \in \mathrm{GL}_{n}(\mathbf{R})\right\}$. Define the bilinear form $\varphi$ on the column space $V=\mathbf{R}^{n}$ by $\varphi(x, y)=x^{\prime} A y$. Let $m(r)$ be the multiplicity in $(V, \varphi)$ of the Gabriel block $N_{r}$, as defined prior to Theorem 3.3. Let $B$ be the matrix of $V_{\text {ndeg }}$ relative to some basis, and set $S=B^{-1} B^{\prime}$. Then $O_{A}$ is disconnected if and only if $m(r)=0$ for odd $r$ 's and $S$ has no elementary divisors of the form $(t-1)^{d}$ with $d$ odd.

Let us make three remarks that are useful for the applications of this theorem.

1) If $\operatorname{det}\left(A-A^{\prime}\right) \neq 0$, then $O_{A}$ is disconnected (see the remark following (4.1)).

2) If $A$ is nonsingular and $S=A^{-1} A^{\prime}$, then the number of unipotent Jordan blocks of $S$ is equal to the nullity of $A-A^{\prime}$. This follows from the equality $I_{n}-S=$ $A^{-1}\left(A-A^{\prime}\right)$.

3) If $O_{A}$ is disconnected, then $\operatorname{dim} V, \operatorname{dim} V_{\text {ndeg }}$ and $\operatorname{dim} V_{\text {tdeg }}$ must be even.

As an example, we shall apply the above theorem to determine for which real square matrices $A$, of size $n \leq 4$, the congruence class $O_{A}$ is disconnected. Of course, this can only occur for $n=2$ or $n=4$. In the former case exactly the non-symmetric $A$ will have disconnected orbit.

Now let $n=4$ and let $V=\mathbf{R}^{4}$ and $\varphi$ be as in the theorem. 
If $V=V_{\mathrm{tdeg}}$, then $O_{A}$ is disconnected if and only if $A$ is congruent to $J_{4}$ or $J_{2} \oplus J_{2}$.

Next let $V_{\text {ndeg }}$ and $V_{\text {tdeg }}$ each have dimension 2. Then we may assume that $A=J_{2} \oplus B$, where $\operatorname{det}(B) \neq 0$. The orbit $O_{A}$ will be disconnected if and only if $B$ is not symmetric.

Finally let $A$ be non-singular and $S=A^{-1} A^{\prime}$. Clearly, $O_{A}$ is connected if $A-A^{\prime}$ has rank 0 and disconnected if this rank is 4 . Thus assume that its rank is 2 . Then we may assume that

$$
A=\left(\begin{array}{cc}
B+K & C \\
C^{\prime} & D
\end{array}\right), \quad K=\left(\begin{array}{rr}
0 & 1 \\
-1 & 0
\end{array}\right)
$$

where $B$ and $D$ are symmetric matrices of size 2 . If $D \neq 0$ it is easy to see that $V$ has a 1-dimensional orthogonal summand and so $O_{A}$ is connected. We may now assume that $D=0$ and so $C$ is invertible. It is now easy to see that $A$ is congruent to

$$
\left(\begin{array}{cccc}
0 & 1 & 1 & 0 \\
-1 & 0 & 0 & 1 \\
1 & 0 & 0 & 0 \\
0 & 1 & 0 & 0
\end{array}\right) .
$$

In this case the two unipotent Jordan blocks of $S$ each have size 2 and so $O_{A}$ is disconnected.

This last case can be easily generalized to obtain the following interesting

Corollary 5.2. If $n$ is divisible by 4 , say $n=4 k$, then there exists a unique disconnected congruence class $O_{A} \subseteq M_{n}(\mathbf{R})$ such that $A-A^{\prime}$ has rank $2 k$. Such $A$ is congruent to the direct sum of $k$ copies of the matrix (5.1), which is itself indecomposable.

\section{REFERENCES}

[1] C.S. Ballantine and E.L. Yip, Congruence and conjunctivity of matrices, Linear Algebra Appl., 32 (1980), 159-198. MR0577914 (81f:15017)

[2] B. Corbas and G.D. Williams, Congruence classes in $M_{3}\left(F_{q}\right)$ ( $q$ even), Discrete Math., 257 (2002), 15-27. MR1931489 (2003i:15013)

[3] B. Corbas and G.D. Williams, Congruence classes in $M_{3}\left(F_{q}\right)$ ( $q$ odd), Discrete Math., 219 (2000), 37-47. MR1761709 (2001e:15007)

[4] B. Corbas and G.D. Williams, Bilinear forms over an algebraically closed field, J. Pure Appl. Algebra, 165 (2001), 255-266. MR1864472 (2002i:15028)

[5] J. Dieudonné, Sur les groupes classiques, Troisième édition, Hermann, Paris, 1973. MR0344355 (49:9094)

[6] J. Dieudonné, La géométrie des groupes classiques, Troisième édition, Ergebnisse der Mathematik und ihrer Grenzgebiete, Band 5, Springer-Verlag, Berlin-New York, 1971. MR0310083 (46:9186)

[7] D.Z. Đokovcić and F. Szechtman, An elementary proof of Gabriel's theorem on degenerate bilinear forms and its generalization, J. Algebra, 279 (2004), no. 1, 121-125. MR.2078389

[8] P. Gabriel, Appendix: Degenerate bilinear forms, J. Algebra, 31 (1974), 67-72. MR0347868 $(50: 369)$

[9] R. Gow, The number of equivalence classes of nondegenerate bilinear and sesquilinear forms over a finite field, Linear Algebra Appl., 41 (1981), 175-181. MR0649725 (83k:10042)

[10] R. Gow, The equivalence of an invertible matrix to its transpose, Linear and Multilinear Algebra, 8 (1980), 329-336. MR 0572169 (82f:15008)

[11] A.J. Hahn and O.T. O'Meara, The classical groups and K-theory, Grundlehren der Mathematischen Wissenschaften, 291. Springer-Verlag, Berlin, 1989. MR1007302 (90i:20002) 
[12] J. Lee and D. Weinberg, A note on canonical forms for matrix congruence, Linear Algebra Appl., 249 (1996), 207-215. MR1417418 (97i:15013)

[13] J. Milnor and D. Husemoller, Symmetric Bilinear Forms, Ergebnisse der Mathematik und ihre Grenzgebiete, Band 73, Springer-Verlag, New York, 1973. MF 0506372 (58:22129)

[14] C. Riehm, The equivalence of bilinear forms, J. Algebra, 31 (1974), 44-66. MR0347867 (50:368)

[15] R. Scharlau, Zur Klassifikation von Bilinearformen und von Isometrien über Körpern, Math. Z., 178 (1981), 359-373. MR0635205 (83d:10026)

[16] W. Scharlau, Quadratic and Hermitian Forms, Grundlehren der mathematischen Wissenschaften 270, Springer-Verlag, New York, 1985. MR0770063 (86k:11022)

[17] G.E. Wall, On the conjugacy classes in the unitary, symplectic and orthogonal groups, J. Austral. Math. Soc., 3 (1963), 1-62. MR0150210(27:212)

[18] W.C. Waterhouse, The number of congruence classes in $M_{n}\left(\mathbf{F}_{q}\right)$, Finite Fields and their Applications, 1 (1995), 57-63. MR1334625 (96c:11141)

[19] J. Williamson, On the algebraic problem concerning the normal forms of linear dynamical systems, Amer. J. Math., 58 (1936), 141-163.

[20] R.J. Zimmer, Ergodic Theory and Semisimple Groups, Birkhäuser, Boston, 1984. MR0776417 (86j:22014)

Department of Pure Mathematics, University of Waterloo, Waterloo, Ontario, CANADA N2L 3G1

E-mail address: djokovic@uwaterloo.ca

Department of Mathematics and Statistics, University of Regina, Regina, SaskaTCHEWAN, CANADA S4S 0A2

E-mail address: szechtf@math.uregina.ca 